\title{
Evolutionary Outcomes of Diversely-Functionalized Aptamers Isolated from In Vitro Evolution
}

\section{Supporting information}

Dehui Kong, ${ }^{\dagger}$ Matina Movahedi, ${ }^{\S}$ Yasaman Mahdavi-Amiri, ${ }^{\S}$ Wayland Yeung, ${ }^{\dagger}$ Tristan R. Tiburcio, ${ }^{\S}$ Dickson Chen, ${ }^{\S}$ and Ryan Hili ${ }^{*+, \S}$

${ }^{+}$Department of Chemistry, University of Georgia, 140 Cedar Street, Athens, GA 30602, USA

${ }^{\circledR}$ Department of Chemistry, York University, 4700 Keele Street, Toronto, ON M3J 1P3, Canada

\section{Supporting Information}

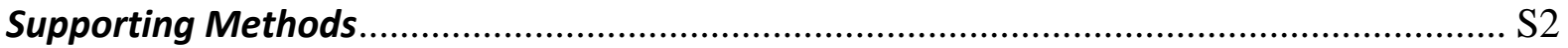

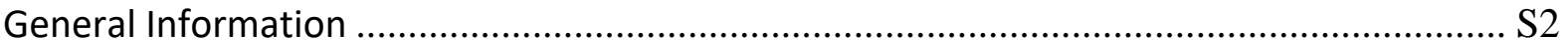

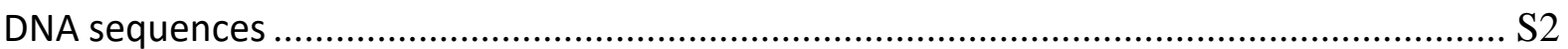

Synthesis of amino-modified pentanucleotides ............................................................... S3

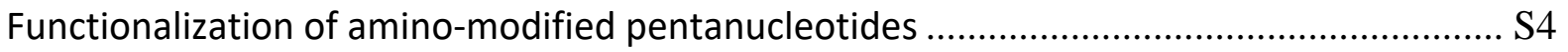

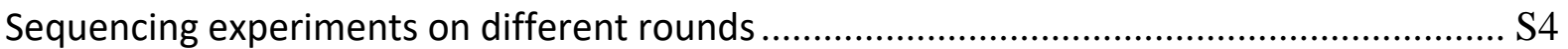

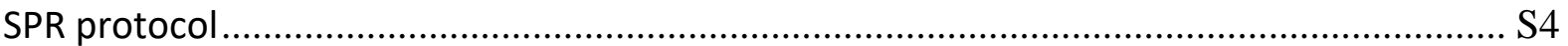

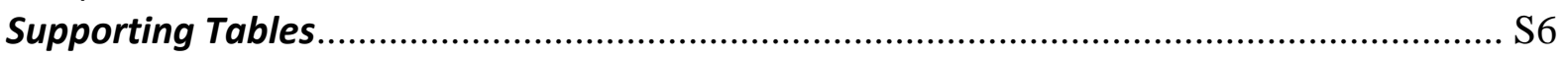

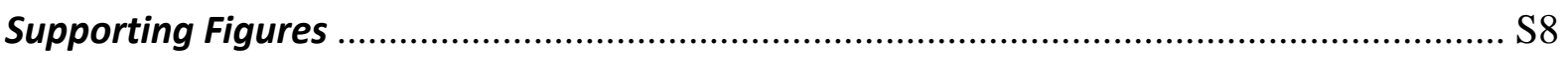




\section{Supporting Methods}

\section{General Information}

Unless otherwise noted, water was purified with the ELGA Flex 3 purification system. DNA oligonucleotides without amine modifications were purchased from Integrated DNA Technologies. DNA oligonucleotides with amine modifications were synthesized using a Bioautomation Mermade 12 synthesizer. All materials and reagents used for oligonucleotide synthesis were purchased from Glen Research. All oligonucleotides were synthesized and deprotected according to the manufacturer's protocols. Oligonucleotides were purified by reverse-phase high-performance liquid chromatography (HPLC, Agilent 1260) using a C18 stationary phase (Eclipse-XDB C18, $5 \mu \mathrm{m}, 9.4 \times 200 \mathrm{~mm}$ ) and an acetonitrile/100 mM triethylammonium acetate gradient. Oligonucleotide concentrations were determined by UV spectroscopy using a Nanodrop ND2000 spectrophotometer. Thrombin agarose slurry (EMD Millipore $^{\mathrm{TM}}$ Thrombin, Immobilized, Human Plasma, Protein) was purchased from EMD Millipore $^{\mathrm{TM}}$. Thrombin (HCT-0020 Human alpha-Thrombin) used for MST and SPR was purchased from Haematologic Technologies.

Valeric acid (Sigma-Aldrich, cat.no.240370), Cyclopropylacetic acid (Sigma-Aldrich, cat.no.CDS000888), 1H-Tetrazole-5-acetic acid (Sigma-Aldrich, cat.no.544108), Succinamic acid (Sigma-Aldrich, cat.no.134376), Isovaleric acid (Sigma-Aldrich, cat.no.129542), Isobutyric acid (Sigma-Aldrich, cat.no.I1754), 3,4-(Methylenedioxy)phenylacetic acid (Sigma-Aldrich, cat.no.329673), Phenylacetic acid (Sigma-Aldrich, cat.no.P16621), Succinic anhydride (SigmaAldrich, cat.no.239690), Cyclopentylacetic acid(Sigma-Aldrich, cat.no.125490), 3Thiopheneacetic acid (Sigma-Aldrich, cat.no.220639), 1-methyl-4-piperidinecarboxylic acid (Sigma-Aldrich, cat.no. PH009778), Glycolic acid (Sigma-Aldrich, cat.no.124737), Nicotinic acid (Sigma-Aldrich, cat.no.N4126) and Succinic anhydride (Sigma-Aldrich, cat.no.239690) were purchased from Sigma-Aldrich.

\section{DNA sequences}

The sequences below are written from $5^{\prime} \rightarrow 3^{\prime}$

$<$ Aam $>=$ Amino-modifer $\mathrm{C} 6 \mathrm{dA}$

$<\mathrm{N}>=\mathrm{A} / \mathrm{T} / \mathrm{C} / \mathrm{G}$

SSDNA

LOOPERtemp: /5BioTinTEG/GA TTC GCC TGC CGT CGC ANN NNT NNN NTN NNN TNN NNT NNN NTN NNN TNN NNT NNN NTC ACG TGG AGC TCG GAT CC

Antisense-TBL1: GAT TCG CCT GCC GTC GCA AGC ATC GAA TAG GAT TGG ATA GGT TGG AAT TAG ATG CAG TCA CGT GGA GCT CGG ATC C

Bt-antisenseTBL1: /5BioTinTEG/GA TTC GCC TGC CGT CGC AAG CAT CGA ATA GGA TTG GAT AGG TTG GAA TTA GAT GCA GTC ACG TGG AGC TCG GAT CC

UnmodTBL1: GGA TCC GAG CTC CAC GTG ACT GCA TCT AAT TCC AAC CTA TCC AAT CCT ATT CGA TGC TTG CGA CGG CAG GCG AAT C

UnmodTBL1_3idT: GGA TCC GAG CTC CAC GTG ACT GCA TCT AAT TCC AAC CTA TCC AAT CCT ATT CGA TGC TTG CGA CGG CAG GCG AAT C/3InvdT/

Pr_3idT: /5Phos/TG CGA CGG CAG GCG AAT C/3InvdT/

TBA: GGTTGGTGTGGTTGG 
HD22: AGTCCGTGGTAGGGCAGGTTGGGGTGACT

Poly_pr1: /5Phos/TG CGA CGG CAG GCG AAT C

Poly_pr2: GGA TCC GAG CTC CAC GTG

bt-prA: /5BioTinTEG/GA TTC GCC TGC CGT CGC A

PEG-prB: AAC AAC AAC AAC AA/iSp18/ GGA TCC GAG CTC CAC GTG

bt-prB: /5BioTinTEG/ GG ATC CGA GCT CCA CGT G

prD701: CAA GCA GAA GAC GGC ATA CGA GAT ATT ACT CG GTGACTGGAGTTCAG ACG TGT

GCT CTT CCG ATCT GA TTC GCC TGC CGT CGC A

prD702: CAA GCA GAA GAC GGC ATA CGA GAT TCC GGA GA GTGACTGGAGTTCAG ACG TGT

GCT CTT CCG ATCT GA TTC GCC TGC CGT CGC A

prD703: CAA GCA GAA GAC GGC ATA CGA GAT CGC TCA TT GTGACTGGAGTTCAG ACG TGT

GCT CTT CCG ATCT GA TTC GCC TGC CGT CGC A

prD704: CAA GCA GAA GAC GGC ATA CGA GAT CGC TCA TT GTGACTGGAGTTCAG ACG TGT

GCT CTT CCG ATCT GA TTC GCC TGC CGT CGC A

prD705: CAA GCA GAA GAC GGC ATA CGA GAT ATT CAG AA GTGACTGGAGTTCAG ACG TGT

GCT CTT CCG ATCT GA TTC GCC TGC CGT CGC A

prC: AAT GAT ACG GCG ACC ACC GAG ATC TAC ACT CTT TCC CTA CAC GAC GCT CTT CCG ATC

TGG ATC CGA GCT CCA CGT G

dsDNA

dsDNA TBL1: GGA TCC GAG CTC CAC GTG ACT GCA TCT AAT TCC AAC CTA TCC AAT CCT ATT CGA TGC TTG CGA CGG CAG GCG AAT C

(dsDNA TBL1 sequence is the same as SSDNA TBL1)

\section{Synthesis of amino-modified pentanucleotides}

Pentanucleotides were synthesized on a Mermaid 12 DNA synthesizer using a DMT-ON protocol on a $1 \mu \mathrm{mol}$ scale (1000 Å CPG column). Amine-modified C6 dA (Glen Research 101089), dA+dC+dG+dT-CE Phosphoramindite (Glen Research 10-1000, 10-1010, 10-1020, 101030), Chemical Phosphorylation Reagent II (10-1901) were incorporated as specified by the manufacturer. Following synthesis, the oligonucleotide was cleaved from the resin by incubation at $25^{\circ} \mathrm{C}$ in $400 \mu \mathrm{L}$ of a $1: 1$ mixture of ammonium hydroxide and methylamine for 25 minutes. The cleaved resin was filtered away, then incubated at $60^{\circ} \mathrm{C}$ for 30 minutes to remove the protecting groups on the phosphoramidites. The oligonucleotide was concentrated under reduced pressure using a speedvac. The residue was then taken up into $100 \mu \mathrm{L}$ of $\mathrm{H}_{2} \mathrm{O}$, and purified using reverse-phase HPLC purification using a [10\% acetonitrile in $0.1 \mathrm{M}$ TEAA, $\mathrm{pH}$ 7] to [ $80 \%$ acetonitrile in $0.1 \mathrm{M} \mathrm{TEAA}, \mathrm{pH}$ 7] solvent gradient with a column temperature of $45^{\circ} \mathrm{C}$. The purified oligonucleotide was then incubated at room temperature in $1 \mathrm{~mL}$ of $40 \%$ aqueous acetic acid for 1 hour to cleave the DMT group, and then frozen and lyophilized. The oligonucleotide was incubated in $500 \mu \mathrm{L} 30 \%$ ammonium hydroxide at room temperature for 15 minutes to cleave the CPRII linker. Following deprotection, the oligonucleotide was concentrated under reduced pressure using a speedvac. The dried product was dissolved into $100 \mu \mathrm{L} \mathrm{H}_{2} \mathrm{O}$ and subjected to reverse-phase HPLC purification using a [10\% acetonitrile in $0.1 \mathrm{M} \mathrm{TEAA}, \mathrm{pH}$ 7] to [80\% acetonitrile in $0.1 \mathrm{M} \mathrm{TEAA}, \mathrm{pH}$ 7] solvent gradient with a column temperature of $45^{\circ} \mathrm{C}$. The purified oligonucleotide was dissolved in water. 


\section{Functionalization of amino-modified pentanucleotides}

A mixture of $25 \mu \mathrm{L}$ carboxylic acid ( $100 \mathrm{mM}$ in DMSO), $25 \mu \mathrm{L}$-hydroxysuccinimide (NHS, 100 $\mathrm{mM}$ in 1:1 mixture of DMSO and $\mathrm{H}_{2} \mathrm{O}$ ), $5 \mu \mathrm{L}$ 1-Ethyl-3-(3-dimethylaminopropyl) carbodiimide (EDC, $100 \mathrm{mM}$ in DMSO) and 7.5 $\mu \mathrm{L}$ DMSO was incubated at room temperature for 30 minutes. This is followed by addition $7.5 \mu \mathrm{L}$ of amino modified pentanucleotides ( $10 \mathrm{nmol}$ in $\mathrm{H}_{2} \mathrm{O}$ ) and $30 \mu \mathrm{L} \mathrm{Na}{ }_{2} \mathrm{CO}_{3}$ buffer $\left(500 \mathrm{mM}\right.$ in $\mathrm{H}_{2} \mathrm{O}, \mathrm{pH}$ 9). The mixture was incubated at room temperature

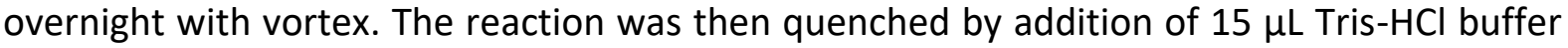
( $500 \mathrm{mM}, \mathrm{pH} 8$ in $\mathrm{H}_{2} \mathrm{O}$ ) at room temperature for one hour. Functionalized pentanucleotide was lyophilized and then purified with HPLC purification using a [10\% acetonitrile in $0.1 \mathrm{M}$ TEAA, $\mathrm{pH}$ 7] to [ $80 \%$ acetonitrile in $0.1 \mathrm{M} \mathrm{TEAA}, \mathrm{pH}$ 7] solvent gradient with a column temperature of $45^{\circ} \mathrm{C}$. The purified pentanucleotide was dissolved in water and characterized by mass spectrometry.

The acid-modified pentamer ( $\left.\mathrm{A}^{\times \mathrm{R}} \mathrm{NNCT}\right)$ is synthesized with an anhydride instead of a carboxylic acid. A mixture of $25 \mu \mathrm{L}$ succinic annhydride (100 mM in DMSO), $25 \mu \mathrm{L} 1 \mathrm{M} \mathrm{NaHCO}_{3}$, $163 \mu \mathrm{L}$ DMSO, $22.4 \mu \mathrm{L}$ water and $14.6 \mu \mathrm{L}$ of amino modified pentanucleotides $(25 \mathrm{nmol}$ in $\mathrm{H}_{2} \mathrm{O}$ ) was incubated at room temperature overnight with vortex. The reaction was then quenched by addition of $50 \mu \mathrm{L}$ Tris- $\mathrm{HCl}$ buffer $\left(500 \mathrm{mM}, \mathrm{pH} 8\right.$ in $\left.\mathrm{H}_{2} \mathrm{O}\right)$ at room temperature for one hour. Functionalized pentanucleotide was lyophilized and then purified with HPLC purification using a [ $10 \%$ acetonitrile in $0.1 \mathrm{M} \mathrm{TEAA}, \mathrm{pH}$ 7] to [ $80 \%$ acetonitrile in $0.1 \mathrm{M} \mathrm{TEAA}$, $\mathrm{pH}$ 7] solvent gradient with a column temperature of $45^{\circ} \mathrm{C}$. Each of the 16 pentanucleotides was dissolved to $480 \mu \mathrm{M}$. Mixtures were assembled with equal volumes of each pentanucleotide.

\section{Sequencing experiments on different SELEX rounds}

Every round selection winners were qPCR amplified with bt-prA and PEG-prB during the SELEX process. Barcode primers (prD705 and prC) were attached by another qPCR amplification. A mixture of 50 amol purified adapter ligation product in $10 \mu \mathrm{L} \mathrm{H} 2 \mathrm{O}, 1.25 \mu \mathrm{L} 10 \mu \mathrm{M}$ prC, 1.25 $\mu \mathrm{L} 10 \mu \mathrm{M}$ corresponding prD701 prD705 primer, and $12.5 \mu \mathrm{L}$ Q5 ${ }^{\circledR}$ High-Fidelity $2 \times$ Master Mix (New England Biolabs) was transferred to a preheated themocycler $\left(98^{\circ} \mathrm{C}\right)$. The first two PCR cycles were 10 seconds at $98^{\circ} \mathrm{C}$ (denaturation), 30 seconds at $55^{\circ} \mathrm{C}$ (annealing), and 30 seconds at $72{ }^{\circ} \mathrm{C}$ (extension). From Cycle3, annealing was changed to $71{ }^{\circ} \mathrm{C}$ for 30 seconds. The PCR products were then gel purified. The concentrations of gel purified samples were determined with Kapa library quantification kit for Illumina libraries (KK4845) on a Roche LightCycler 480. Paired-end Illumina sequencing was performed on an Illumina MiSeq system using the kit v2 with 300 cycles (150 bp PE sequencing) at the Georgia Genomics Facility at The University of Georgia in Athens, GA, USA.

\section{SPR protocol}

A Biacore T100 and Biotin CAPture Kit (GE Healthcare Life Sciences) were used in SPR interaction studies ${ }^{4}$. Sensor chip CAP was conditioned and regenerated according to manufacturer's specifications before initial use. Sensor chip CAP consists of a carboxymethylated dextran matrix modified with a pre-immobilized oligonucleotide. Complementary oligonucleotide conjugated with streptavidin (Biotin CAPture Reagent) was added later to build a streptavidin surface with an increase of $\sim 3000 \mathrm{RU}$. A new sensor chip CAP would be used if the capture capacity (RU levels) decreased significantly. A reference channel was also built with streptavidin surface according to Biotin CAPture Kit specifications. 
$50 \mathrm{nM}$ biotinylated TBL3 and deletion mutants modTBL1 in running buffer (identical to SELEX Buffer) were added at flow rate of $5 \mu \mathrm{L} / \mathrm{min}$ for 300 seconds with a net increase of 100 200 RU. Thrombin was serially diluted in running buffer to a concentration range of $5 / 10 / 20 / 30 / 50 \mathrm{nM}$ and injected at $21^{\circ} \mathrm{C}$ at a flow rate of $5 \mu \mathrm{L} / \mathrm{min}$ for 300 seconds and dissociated for 600 seconds. Single-cycle-kinetics mode ${ }^{5}$ was used with thrombin injection from lowest concentration to highest concentration. Surface regeneration at the conclusion of each session as per Biotin CAPture Kit specifications. Due to yield limitations and purification loss in LOOPER, $\triangle 6$ _TBL1 yield was slightly less than the others. $5 \mathrm{nM}$ biotinylated $\Delta 6 \_$TBL1 were added at flow rate $5 \mu \mathrm{L} / \mathrm{min}$ for 300 seconds with a net increase of $\sim 25 \mathrm{RU}$. Thrombin was serially diluted in running buffer to a concentration range of $0.5 / 2 / 5 / 10 / 20 \mathrm{nM}$ and injected at $21^{\circ} \mathrm{C}$ at a flow rate of $5 \mu \mathrm{L} / \mathrm{min}$ for 300 seconds and dissociated for 600 seconds 


\section{Supporting Tables}

Table S1. Hamming distance matrix among Round6 SELEX hits.

\begin{tabular}{c|lllll} 
& TBL1 & TBL2 & TBL3 & TBL4 & TBL5 \\
\hline TBL1 & 0 & 14 & 11 & 17 & 19 \\
TBL2 & 14 & 0 & 20 & 16 & 15 \\
TBL3 & 11 & 20 & 0 & 16 & 23 \\
TBL4 & 17 & 16 & 16 & 0 & 19 \\
TBL5 & 19 & 15 & 23 & 19 & 0
\end{tabular}

Table S2. Error filter pipelines of NGS data.

\begin{tabular}{cccc}
\hline & Round 2 & Round 4 & Round 6 \\
\hline Total reads & $1,893,631$ & 668,807 & 888,634 \\
Wrong primers & 35,905 & 17,278 & 33,192 \\
Primer error rate & $1.90 \%$ & $2.58 \%$ & $3.74 \%$ \\
Wrong length & 20 & 6 & 18 \\
Length error rate & $<0.01 \%$ & $<0.01 \%$ & $<0.01 \%$ \\
Not ANNNN pattern & 52,078 & 19,012 & 4,740 \\
LOOPER error rate & $2.75 \%$ & $2.84 \%$ & $0.53 \%$ \\
Total error & 88,003 & 36,296 & 37,950 \\
Total error rate & $4.65 \%$ & $5.43 \%$ & $4.27 \%$ \\
Usable reads & $1,805,628$ & 632,511 & 850,684 \\
\hline
\end{tabular}


Table S3. SELEX hits alignment table in (a) Round 2 , (b) Round 4.

(a)

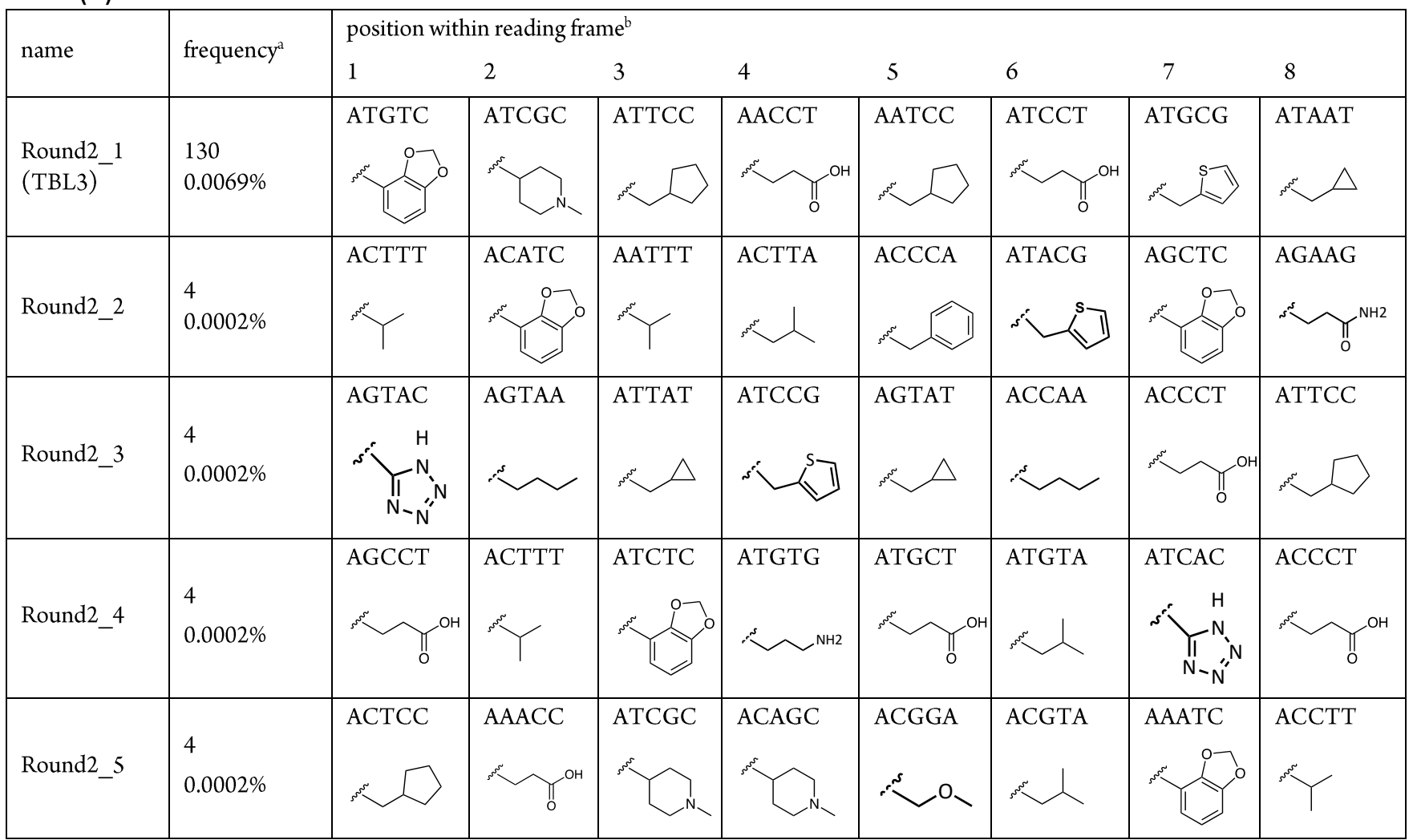

a: reads of unique DNA sequence observed by high-throughput DNA sequencing; total reads $=1,893,631 . \quad b$ : positions listed from $5^{\prime}$ to $3^{\prime}$.

(b)

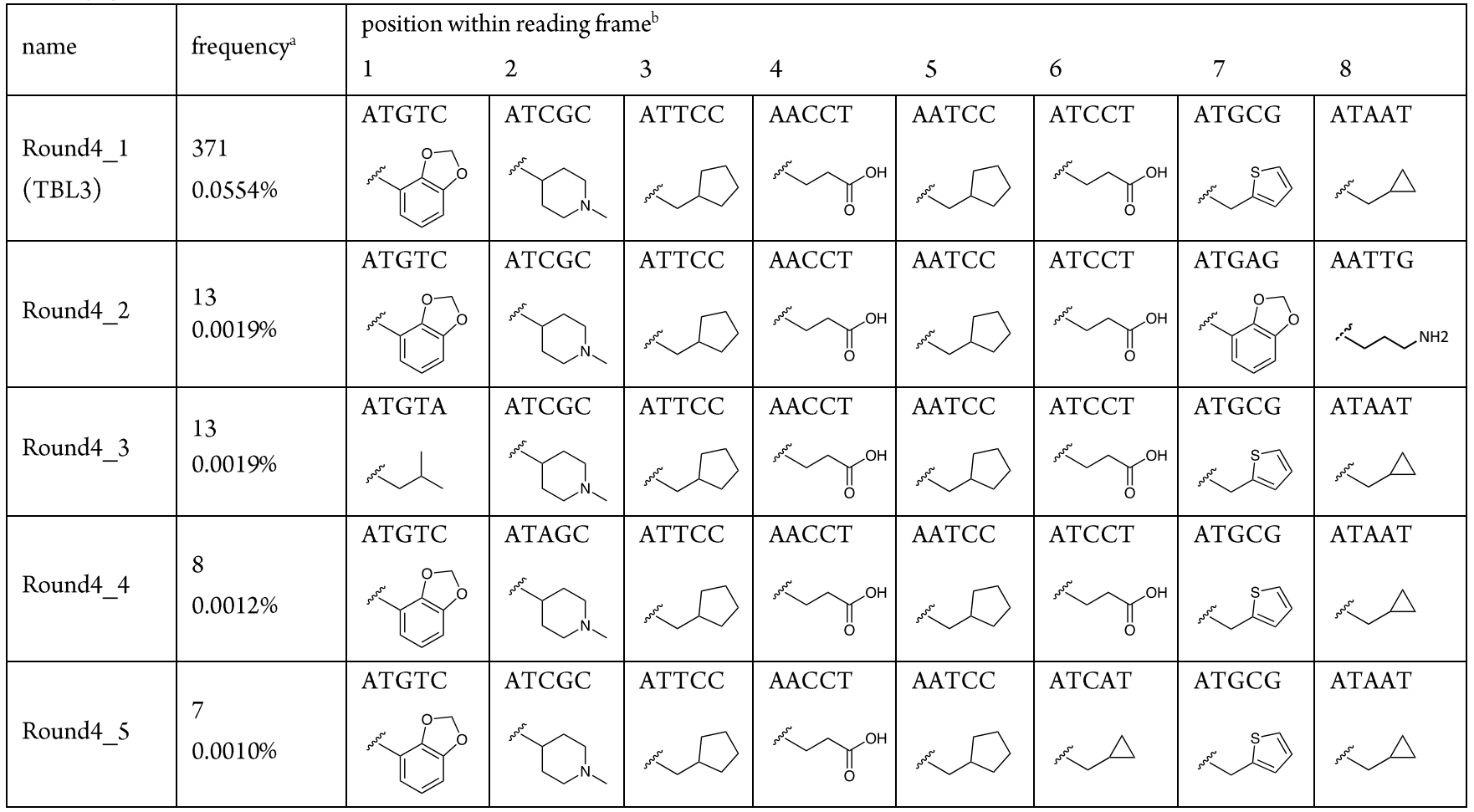




\section{Supporting Figures}
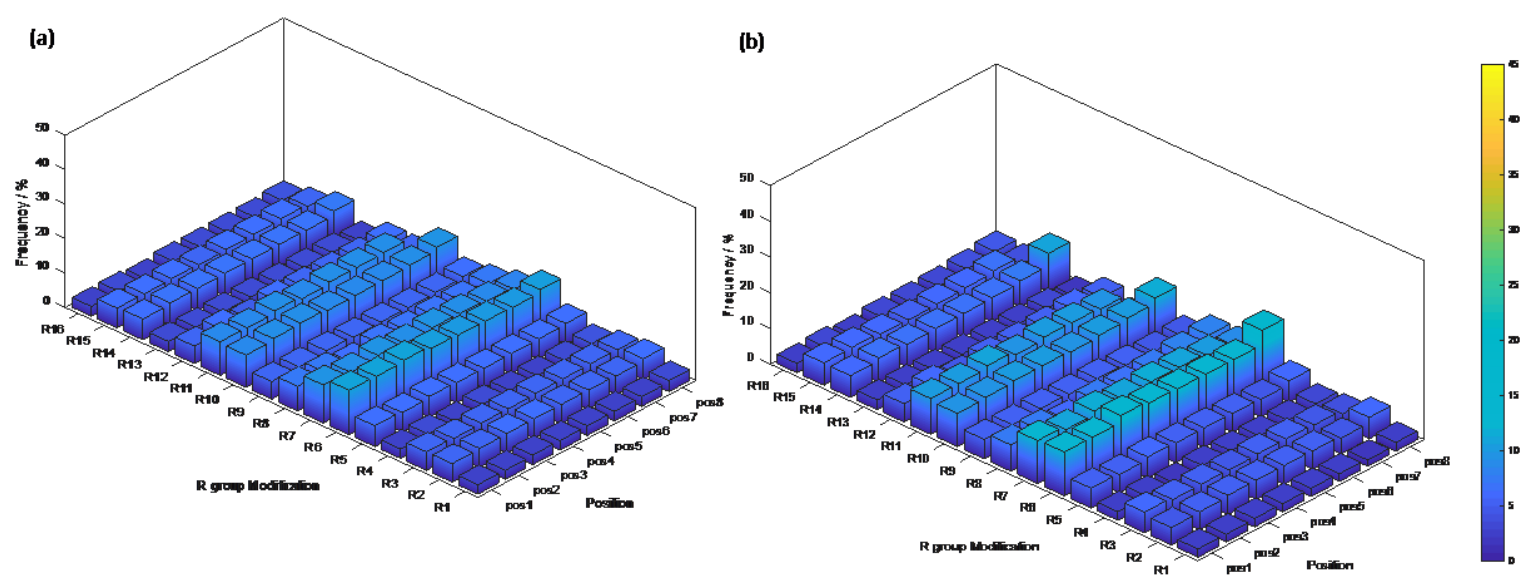

Figure S1. Modification-position barplot for LOOPER-SELEX (a) Round 2 and (b) Round 4. Round 6 information is in Figure 2a.

(a)

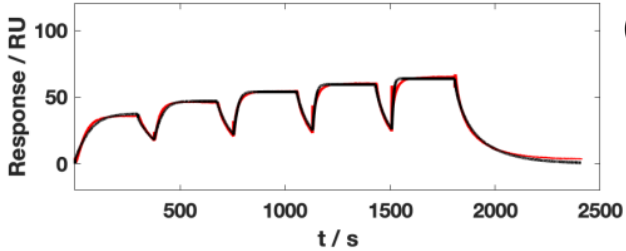

(c)

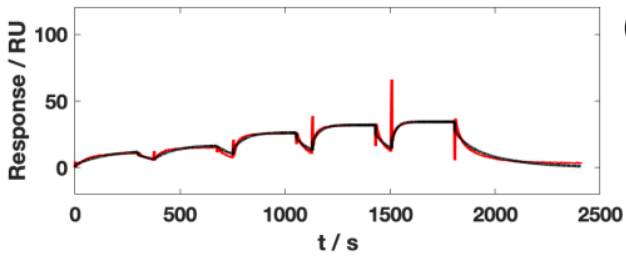

(e)

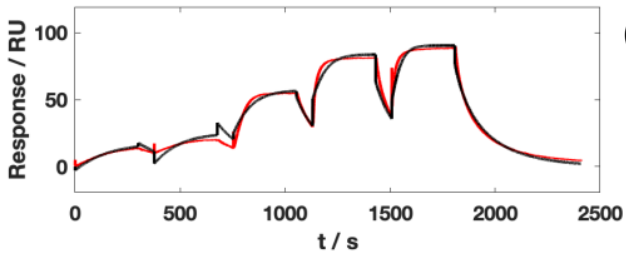

(g)

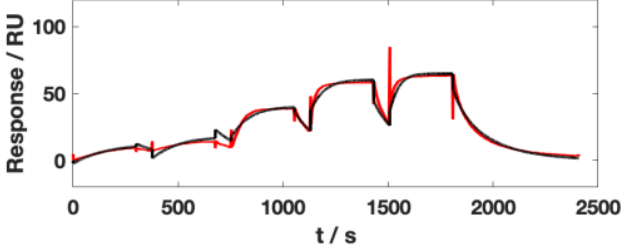

(b)

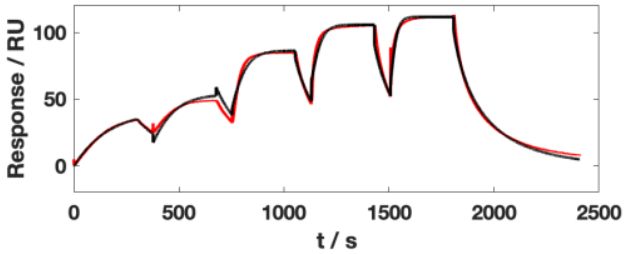

(d)

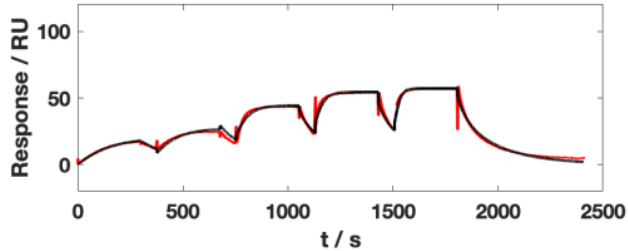

(f)

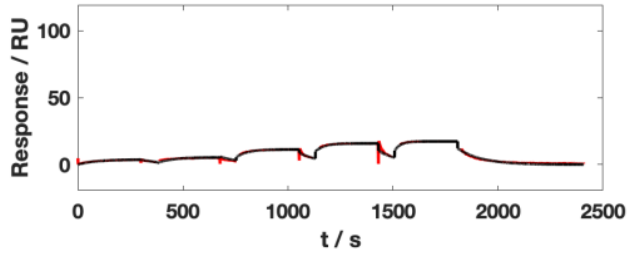

(h)

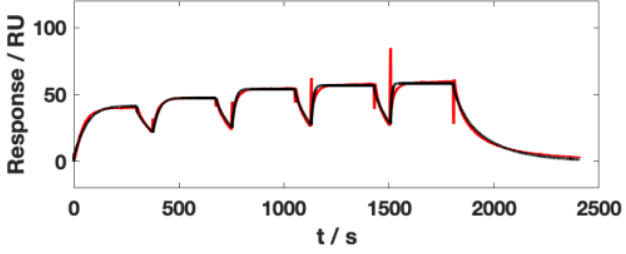

Figure S2. Single cycle kinetics SPR for single modifier knock-out versions of TBL1 binding to thrombin. $\Delta 1$ 1_TBL1 $\sim 8_{-}$TBL1: (a) $\sim$ (h). 


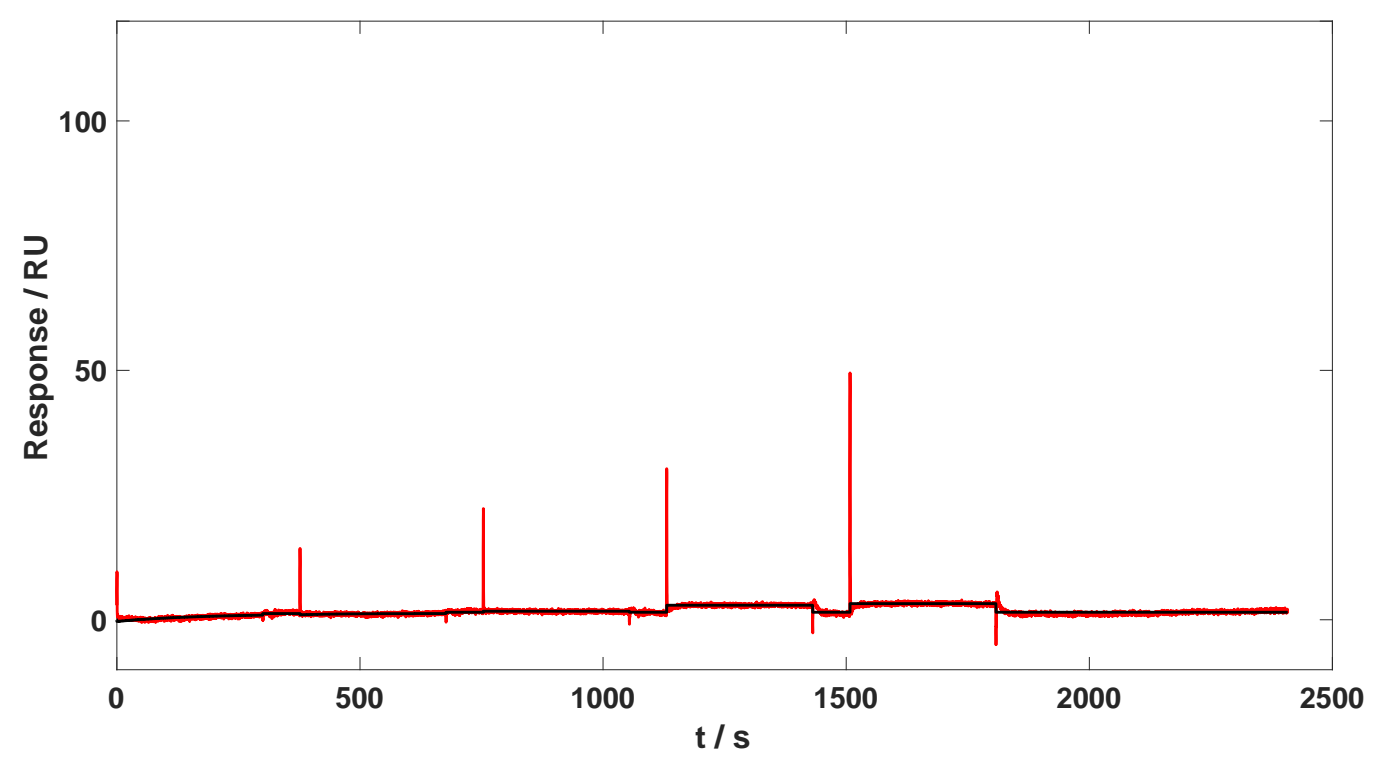

Figure S3. Single cycle kinetics SPR for hairpin modifier deletions of TBL1 ( $\triangle \mathrm{hp} \_$TBL1) binding to thrombin.

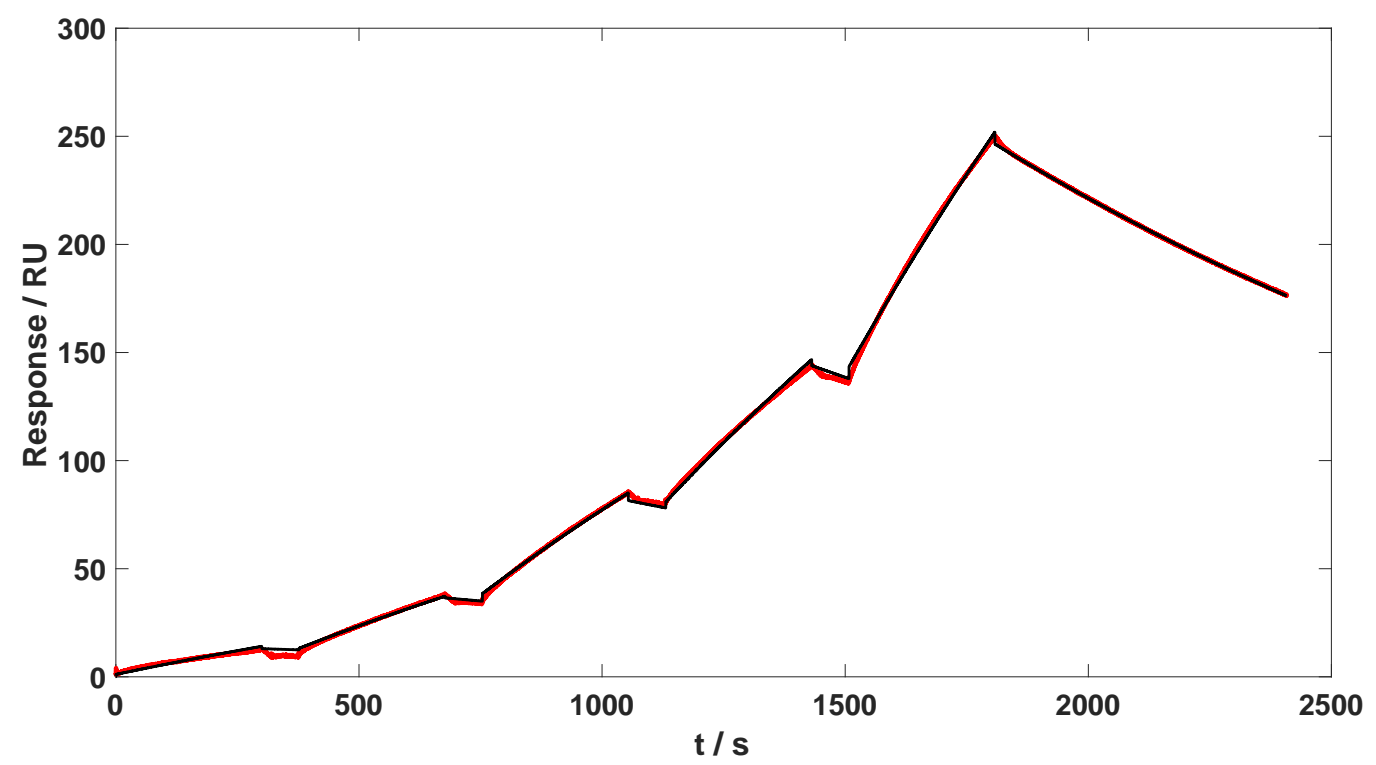

Figure S4. Single cycle kinetics SPR for TBL3 binding to thrombin.
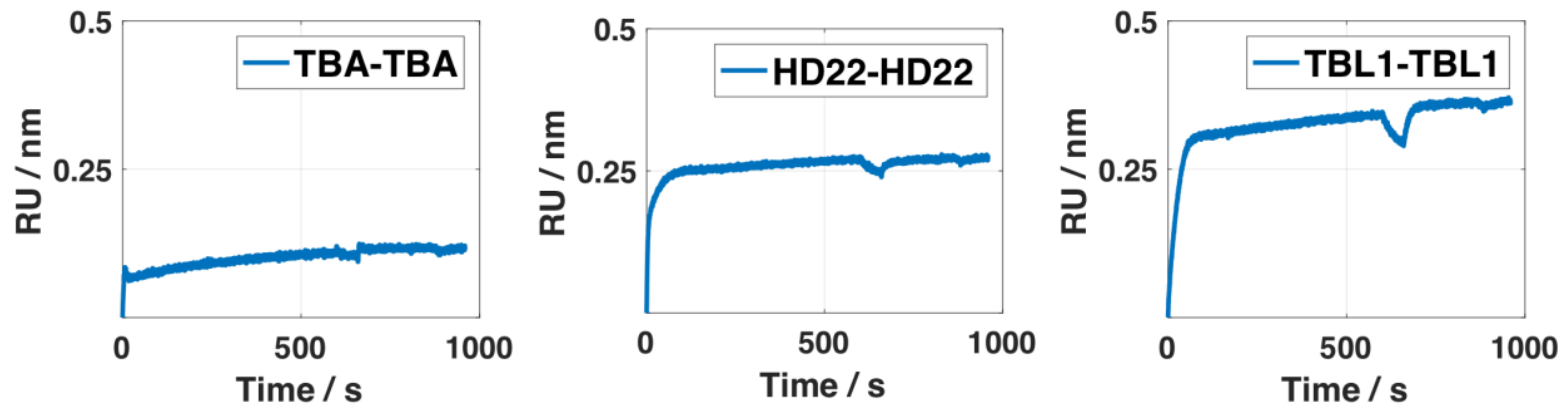

Figure S5. Epitope binning sensorgrams on Biolayer Interferometry (BLI) for self-binning sensorgrams for system validations. 


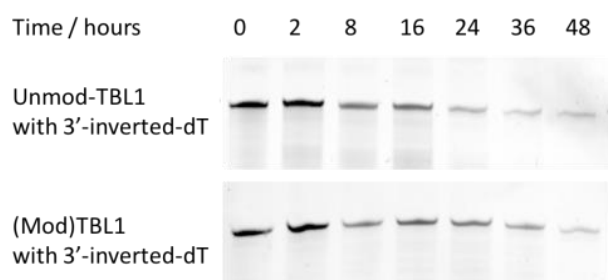

Figure S6. Nuclease resistance analysis. Unmodified-TBL1 and Modified-TBL1 nuclease resistance experiments in 90\% serum for 0-48 hours.

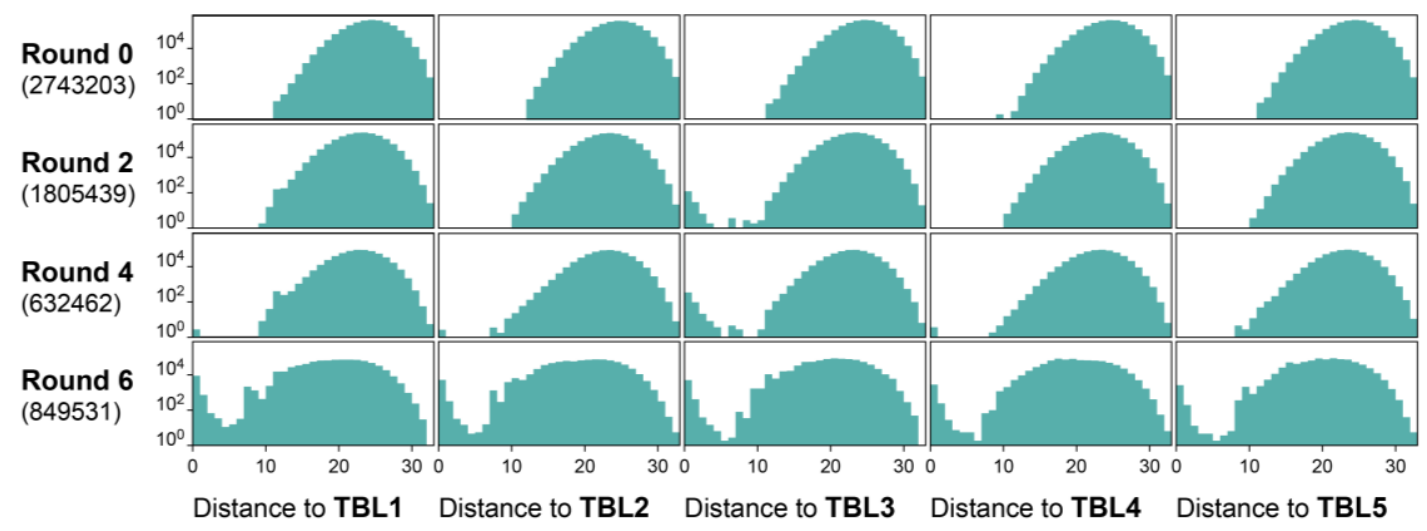

Figure S7. Hamming Distance of R-group modifications for each TBL sequence mapped by using the ANNXX pentanucleotides. 